\title{
PROBABILITY MEASURE REPRESENTATION OF NORMS ASSOCIATED WITH THE NOTION OF ENTROPY
}

\author{
ROMUALD DABROWSKI
}

\begin{abstract}
One of the applications of Banach spaces introduced by B. Korenblum $[1,2]$ is a new convergence test [2] for Fourier series including both Dirichlet-Jordan and the Dini-Lipschitz tests [3]. The norms of the spaces are given in terms of $\kappa$-entropy where $\kappa(s) \geqslant 0,0<s \leqslant 1$, is a nondecreasing concave function such that $\kappa(1)=1$. The $\kappa$-norms fill the gap between the uniform and the variation norms. The original proof of the general properties of $\kappa$-norms uses both combinatorial and approximation arguments which are rather complicated. We give a simple proof introducing a probabilistic representation of the norms so that the $\kappa$-norm of a real function $f$ on $T=R / 2 \pi Z$ is the expectation of the mean oscillation of $f$ on a subinterval of $T$, chosen in a suitable random process.
\end{abstract}

The $\kappa$-entropy. We start with the definition of the $\kappa$-norm introduced by $B$. Korenblum [1,2]. Let $T=R / 2 \pi Z$, and let $|E|=\int_{E} d x$ denote the normalized Lebesgue measure of a Borel subset $E$ of $T$. The distance between points $x, y \in T$ is $d(x, y)=\min \{|x-y+2 \pi n| ; n \in Z\} / 2 \pi . L^{\infty}(T)$ is the space of complex valued essentially bounded function on $T$, and $R L^{\infty}(T)$ denotes the space of real-valued function in $L^{\infty}(T)$. The graph of a function $f \in R L^{\infty}(T)$ is the set

$$
\begin{aligned}
\Gamma(f)=\left\{(t, y) \in T \times R ; \lim _{\delta \rightarrow 0}(\operatorname{essinf}\{f(\tau) ; d(t, \tau)<\delta\}) \leqslant y\right. & \\
& \left.\leqslant \lim _{\delta \rightarrow 0}(\operatorname{ess} \sup \{f(\tau) ; d(t, \tau)<\delta\})\right\} .
\end{aligned}
$$

One shows that $\Gamma(f)$ is always a closed subset of $T \times R$ which is connected on any subinterval of $T$.

DEFINITION 1. Let $\kappa(s), 0<s \leqslant 1$, be a positive nondecreasing concave function such that $\kappa(1)=1$. The $\kappa$-entropy of a finite subset $E$ of $T(E \neq \varnothing)$ is $\kappa(E)=$ $\sum_{i=1}^{n} \kappa\left(\left|I_{i}\right|\right)$ where $\left\{I_{i}\right\}_{i=1}^{n}$ are the complementary intervals of $E$. For an infinite closed subset $E$ of $T(E=\varnothing)$, we set $\kappa(E)=\sup \{\kappa(F) ; F \subset E, F$-finite $\}$. We also put $\kappa(\varnothing)=0$.

DEFINITION 2. For any function $f \in R L^{\infty}(T)$ we set

$$
\|f\|_{\kappa}=\int_{-\infty}^{\infty} \kappa\left(\Gamma_{y}(f)\right) d y
$$

where $\Gamma_{y}(f)=\{t ;(t, y) \in \Gamma(f)\}$ is the $y$-level set of the graph of $f$.

Received by the editors May 23, 1983.

1980 Mathematics Subject Classification. Primary 42A20, 46E15. 
Notice that for a continuous real function $f$ on $T$,

$$
\|f\|_{\kappa}=\|f\|_{C}=\max \{f(t) ; t \in T\}-\min \{f(t) ; t \in T\} \quad \text { if } \kappa(s)=s,
$$

and

$$
\begin{aligned}
\|f\|_{\kappa}=\|f\|_{V}=\sup \left\{\sum_{i=1}^{n}\left|f\left(t_{i}\right)-f\left(t_{i-1}\right)\right| ; t_{0}=0<t_{1}<\cdots<t_{n}=2 \pi\right\} & \text { if } \kappa(s)=1 .
\end{aligned}
$$

For an arbitrary function $\kappa$ as in Definition 1 we have $s \leqslant \kappa(s) \leqslant 1$ for $0<s \leqslant 1$ and, consequently, $\|\cdot\|_{C} \leqslant\|\cdot\|_{\kappa} \leqslant\|\cdot\|_{V}$. If $\kappa\left(0^{+}\right)=\lim _{s \rightarrow 0}(s)=\alpha>0$, then $\kappa=\alpha$ $+\beta \kappa_{0}$ where $\alpha+\beta=1$ and $\kappa_{0}\left(0^{+}\right)=0$. In this case $\|\cdot\|_{\kappa}=\alpha\|\cdot\|_{V}+\beta\|\cdot\|_{\kappa_{0}}$.

If $\kappa\left(0^{+}\right)=0$ and $\lim _{s \rightarrow 0}(\kappa(s) / s)=A<\infty$, then $\|\cdot\|_{C} \leqslant\|\cdot\|_{\kappa} \leqslant A\|\cdot\|_{C}$, i.e. $\|\cdot\|_{\kappa}$ is equivalent to the norm $\|\cdot\|_{C}$. We, therefore, assume in what follows that $\kappa\left(0^{+}\right)=0$ and $\lim _{s \rightarrow 0}(\kappa(s) / s)=\infty$. In this case $\|f\|_{\kappa}=\int_{T \times R^{\prime}} \kappa^{\prime}\left(2 d\left(\Gamma_{y}(f), x\right)\right) d x d y$ where $\kappa(s)=\int_{0}^{s} \kappa^{\prime}(t) d t$. It is by no means simple to show that $\|\cdot\|_{\kappa}$ is, in fact, a norm using just Definition 2 (the triangle inequality is hard to establish). We give another representation of $\kappa$-norms so that many properties of these norms follow naturally.

Probability measure representation of $\kappa$-norms. For closed subsets $A, B$ of $T \times R$ we set $\lambda A=\{(t, \lambda y) ;(t, y) \in A\}, A+B=\left\{\left(t, y+y^{\prime}\right) ;(t, y) \in A,\left(t, y^{\prime}\right) \in B\right\}$ and $A B=\left\{\left(t, y y^{\prime}\right),(t, y) \in A,\left(t, y^{\prime}\right) \in B\right\}$. For $t \in T$ and $0 \leqslant s \leqslant \frac{1}{2}$ we define the oscillation of $A$ on the interval $\{\tau ; d(t, \tau) \leqslant s\}$ by the formula

$$
\Omega_{A}(t, s)=\max \{y ;(\tau, y) \in A, d(t, \tau) \leqslant s\}-\min \{y ;(\tau, y) \in A, d(t, \tau) \leqslant s\} .
$$

We also set $\|A\|_{\infty}=\max \{|y|,(t, y) \in A\}$. One easily verifies $\Omega_{\lambda_{A}}=|\lambda| \Omega_{A}$ for any $\lambda \in R, \Omega_{A+B} \leqslant \Omega_{A}+\Omega_{B}, \Omega_{A} \leqslant \Omega_{B}$ for $A \subseteq B$ and $\Omega_{A B} \leqslant\|A\|_{\infty} \Omega_{B}+\|B\|_{\infty} \Omega_{A}$. For a function $f \in R L^{\infty}(T)$ we put $\Omega_{f}=\Omega_{\Gamma(f)}$. The following Lemma follows easily from the properties of $\Omega$ listed above.

Lemma. Let $f, g \in R L^{\infty}(T)$. We then have

(i) $\Omega_{\lambda f}=|\lambda| \Omega_{f}, \lambda \in R$;

(ii) $\Omega_{f+g} \leqslant \Omega_{f}+\Omega_{g}$;

(iii) $\Omega_{f g} \leqslant\|f\|_{\infty} \Omega_{g}+\|g\|_{\infty} \Omega_{f}$;

(iv) if $f$ is continuously differentiable, then $\Omega_{f}(t, s) \leqslant 2 s\left\|f^{\prime}\right\|_{\infty}$.

Definition 3. Let $\mu$ be a probability measure on the unit interval $[0,1]$. The $\mu$-norm of a function $f \in R L^{\infty}(T)$ is

$$
\|f\|_{\mu}=\int_{T \times[0,1]} \Omega_{f}(t, s / 2) d \mu(s) d t,
$$

where the value of $\Omega_{f}(t, s / 2) / s$ at 0 is $\varlimsup_{s \rightarrow 0}\left(\Omega_{f}(t, s / 2) / s\right)$. Notice that if $\mu$ is concentrated at 1 , then $\|\cdot\|_{\mu}=\|\cdot\|_{C}$. If $\mu$ is concentrated at 0 , and $f$ is an absolutely continuous function on $T$, then $\|f\|_{\mu}=\|f\|_{\nu}$. 
THEOREM 1. Let $\kappa(s), 0<s \leqslant 1$, be a positive nondecreasing concave function such that $\kappa\left(0^{+}\right)=0$ and $\lim _{s \rightarrow 0}(\kappa(s) / s)=\infty$. There is a unique probability measure $\mu_{\kappa}$ on $[0,1]$ such that

$$
\|f\|_{\kappa}=\|f\|_{\mu_{\kappa}}=\int_{T \times[0,1]}\left(\Omega_{f} / s\right)(t, s / 2) d t d \mu_{\kappa}(s) .
$$

The map $\kappa \rightarrow \mu_{\kappa}$ gives a one-to-one correspondence between the sets $K_{0}=\{\kappa ; \kappa$ described above $\}$ and $P_{0}=\{\mu ; \mu$ a probability measure on $[0,1]$ such that $\mu(\{0\})=0$, $\left.\int_{0}^{1} \mu(\tau) / \tau^{2} d \tau=\infty\right\}$.

REMARK. If $\mu$ is a probability measure on $[0,1]$ such that $\mu(\{0\})=0$ then the condition $\int_{0}^{1} \mu(\tau) / \tau^{2} d \tau=\infty$ is equivalent to the condition

$$
\lim _{s \rightarrow 0} \frac{1}{s} \int_{0}^{s} \int_{t}^{1} \frac{d \mu(\tau)}{\tau} d t=\infty
$$

For the proof of the theorem we will use this condition rather than the condition $\int_{0}^{1} \mu(\tau) / \tau^{2} d \tau=\infty$

Proof. Let $\kappa \in K_{0}, \kappa^{\prime}(s), 0 \leqslant s \leqslant 1$, is a nonnegative nonincreasing left-continuous function such that $\kappa^{\prime}(0)=\infty$ and $\kappa(s)=\int_{0}^{s} \kappa^{\prime}(t) d t$. We define $\alpha_{\kappa}(z)=$ $\max \left\{s ; \kappa^{\prime}(s) \geqslant z\right\}=\left|\left\{s ; \kappa^{\prime}(s) \geqslant z\right\}\right|$. The function $\alpha_{\kappa}$ has the properties

$$
\alpha_{\kappa}(z)>0 \text { for all } z \geqslant 0,
$$

$(* *) \quad \kappa^{\prime}(s) \geqslant z \quad$ if and only if $s \leqslant \alpha_{\kappa}(z)$ for all $s \in[0,1], z \geqslant 0$,

$(* * *) \quad \kappa^{\prime}(s)=\max \left\{z ; \kappa^{\prime}(s) \geqslant z\right\}=\left|\left\{z ; \alpha_{\kappa}(z) \geqslant s\right\}\right|$.

The measure $\mu_{\kappa}$ is defined by the formula $d \mu_{\kappa}(s)=s d \alpha_{\kappa}^{-1}(s)$ where $\int_{E} d \alpha_{\kappa}^{-1}(s)=$ $\left|\alpha_{\kappa}^{-1}(E)\right|$ for any Borel subset $E$ of $[0,1]$. For any $0<s \leqslant 1$,

$$
\mu_{\kappa}([0, s])=\int_{0}^{s} t d \alpha_{\kappa}^{-1}(t)=\int_{0}^{\infty} \chi_{[0, s]}\left(\alpha_{\kappa}(z)\right) \alpha_{\kappa}(z) d z
$$

hence,

$$
\mu_{\kappa}([0,1])=\int_{0}^{\infty} \alpha_{\kappa}(z) d z=\int_{0}^{\infty}\left|\left\{s ; \kappa^{\prime}(s) \geqslant\right\}\right| d z=\int_{0}^{\infty} \kappa^{\prime}(s) d s=\kappa(1)=1 .
$$

By the dominated convergence theorem $\mu_{\kappa}(\{0\})=\lim _{s \rightarrow 0} \mu_{\kappa}([0, s])=0$. We show that for any function $f \in R L^{\infty}(T),\|f\|_{\kappa}=\|f\|_{\mu_{\kappa}}$,

$$
\begin{aligned}
\|f\|_{\kappa} & =\int_{T \times R} \kappa^{\prime}\left(2 d\left(\Gamma_{y}(f), x\right)\right) d x d y=\int_{T \times R_{+}}\left|\left\{y ; \kappa^{\prime}\left(2 d\left(\Gamma_{y}(f), x\right)\right) \geqslant z\right\}\right| d x d z \\
& =\int_{T \times R_{+}}\left|\left\{y ; d\left(\Gamma_{y}(f), x\right) \leqslant \alpha_{\kappa}(z) / 2\right\}\right| d x d z
\end{aligned}
$$

because of the property $(* *)$. Notice that $\left|\left\{y ; d\left(\Gamma_{y}(f), x\right) \leqslant s\right\}\right|=\Omega_{f}(x, s)$. We then get

$$
\|f\|_{\kappa}=\int_{T \times R_{+}} \Omega_{f}\left(x, \alpha_{\kappa}(z) / 2\right) d z d x=\int_{T \times[0,1]} \frac{1}{s} \Omega_{f}(x, s / 2) d \mu_{\kappa}(s) d x,
$$


since $\mu_{\kappa}(\{0\})=0$. We now prove the second part of the theorem. Let $\kappa \in K_{0}$ and $\mu=\mu_{\kappa}$. We know that $\mu$ is a probability measure and $\mu(\{0\})=0$. We set

$$
\kappa_{\mu}(s)=\int_{0}^{s} \int_{t}^{1} \frac{1}{\tau} d \mu(\tau) d t
$$

By property $(* * *)$ and the definition of $\mu=\mu_{\kappa}$,

$$
\kappa_{\mu}(s)=\int_{0}^{s} \int_{t}^{1} d \alpha_{\kappa}^{-1}(\tau) d \tau d t=\int_{0}^{s}\left|\alpha_{\kappa}^{-1}([t, 1])\right| d t=\int_{0}^{s} \kappa^{\prime}(t) d t=\kappa(s) .
$$

This shows that $\mu=\mu_{\kappa} \in P_{0}$ and $\kappa_{\mu_{\kappa}}=\kappa_{\mu}=\kappa$.

Assume now that $\mu \in P_{0}$ and let

$$
\kappa(s)=\kappa_{\mu}(s)=\int_{0}^{s} \int_{t}^{1} \frac{1}{\tau} d \mu(\tau) d t .
$$

One can see that $\kappa \in K_{0}$. We next have $\kappa^{\prime}(s)=\int_{s}^{1} d \mu(\tau) / \tau$, and by property (***), $\kappa^{\prime}(s)=\int_{s}^{1} d \alpha_{\kappa}^{-1}(\tau)=\int_{s}^{1} d \mu_{\kappa}(\tau) / \tau$. Consequently, $\mu=\mu_{\kappa}$ since also $\mu(\{0\})=$ $\mu_{\kappa}(\{0\})=0$. This shows that the map $\mu \rightarrow \kappa_{\mu}$ is the inverse of the map $\kappa \rightarrow \mu_{\kappa}$.

REMARKS. If $\kappa \in K_{0}$ and $\kappa^{\prime}$ is continuous strictly decreasing and $\kappa^{\prime}(1)=0$, then $\alpha_{\kappa}$ is the inverse function of $\kappa^{\prime}$. If $\kappa \in K_{0}$ is twice differentiable and $\kappa^{\prime}$ is strictly decreasing then $d \mu_{\kappa}(s)=\kappa^{\prime}(1) d \delta_{1}(s)-s \kappa^{\prime \prime}(s) d s$ where

$$
\delta_{1}(E)= \begin{cases}1 & \text { if } 1 \in E \\ 0 & \text { if } 1 \in E .\end{cases}
$$

For example, if

(1) $\kappa(s)=s^{\alpha}, 0<\alpha<1\left(\kappa(E)\right.$ is the Lipschitz entropy), then $d \mu_{\kappa}(s)=\alpha d \delta_{1}(s)$ $+\alpha(1-\alpha) s^{\alpha-1} d s$

(2) $\kappa(s)=s(|\log s|+1)\left(\kappa(E)\right.$ is the Shannon entropy), then $d \mu_{\kappa}(s)=d s$;

(3) $\kappa(s)=\left(1+\frac{1}{2}|\log s|\right)^{-1}(\kappa(E)$ is the Dini entropy), then

$$
d \mu_{\kappa}(s)=\frac{1}{2} d \delta_{1}(s)+\frac{1}{2} \frac{|\log s|}{s(1+|\log s| / 2)^{3}} d s .
$$

COROLlaRY. $\|\cdot\|_{\kappa}$ is homogeneous and satisfies the triangle inequality for any $\kappa \in K_{0}$. Moreover, if $f$ is a real continuously differentiable function on $T$, then $\|f\|_{\kappa} \leqslant\left\|f^{\prime}\right\|_{\infty} \kappa\left(\|f\|_{C} /\left\|f^{\prime}\right\|_{\infty}\right)$.

Proof. The first part of the Corollary follows directly from the probability representation of the $\kappa$-norms and from the lemma. For the proof of the second part we let $f$ be a nonconstant differentiable function on $T$, so that $\left\|f^{\prime}\right\|_{\infty}<\infty$. We denote $A=\|f\|_{C}$ and $B=\left\|f^{\prime}\right\|_{\infty}(B \neq 0)$. Using the probability representation of the $\kappa$-norm we obtain

$$
\begin{aligned}
\|f\|_{\kappa} & =\int_{T \times[0,1]} \frac{\Omega_{f}(t, s / 2)}{s} d \mu(s) d t=B \int_{T \times[0,1]} \frac{\Omega_{f / B}(t, s / 2)}{s} d \mu(s) d t \\
& =B \int_{T} \int_{0}^{\infty} \Omega_{f / B}\left(t, \alpha_{\kappa}(y) / 2\right) d y d t
\end{aligned}
$$


where $\alpha_{\kappa}$ is the function defined in the proof of Theorem 1. We notice that $\Omega_{f / B}(t, s / 2)$ is nondecreasing and right-continuous in $s$. We define $\beta_{t}(y)=$ $\min \left\{s ; \Omega_{f / B}(t, s / 2) \geqslant y\right\}$ for $0 \leqslant y \leqslant A / B$. Clearly $\Omega_{f / B}(t, s / 2) \geqslant y$ if and only if $s \geqslant \beta_{t}(y)$. Moreover, $\beta_{t}(y) \geqslant y$ for all $0 \leqslant y \leqslant A / B$. Therefore

$$
\begin{aligned}
\|f\|_{\kappa} & =B \int_{T} \int_{0}^{A / B}\left|\left\{z ; \alpha_{\kappa}(z) \geqslant \beta_{t}(y)\right\}\right| d y d x=B \int_{T} \int_{0}^{A / B} \kappa^{\prime}\left(\beta_{t}(y)\right) d y d t \\
& \leqslant B \int_{T} \int_{0}^{A / B} \kappa^{\prime}(y) d y d t=B \kappa(A / B) .
\end{aligned}
$$

In what follows $\kappa$ is again a function from the set $K_{0}$. We define the following linear spaces with the norm $\|\cdot\|_{\infty}+\|\cdot\|_{\kappa} ; R L_{\kappa}^{\infty}(T)=\left\{f \in R L^{\infty}(T) ;\|f\|_{\kappa}<\infty\right\}$ and $R C_{\kappa}=\left\{f ; f\right.$ is continuous on $\left.T,\|f\|_{\kappa}<\infty\right\}$. The analogous spaces $L_{\kappa}^{\infty}$ and $C_{\kappa}$ of complex valued functions are defined as the complexification of the real spaces $R L_{\kappa}^{\infty}$ and $R C_{\kappa}$, respectively (see, for example, [4]). We will use the probability measure representation of $\kappa$-norms to prove some general properties of $L_{\kappa}^{\infty}$ and $C_{\kappa}$.

THEOREM 2. (a) The spaces $L_{\kappa}^{\infty}$ and $C_{\kappa}$ are Banach algebras with the usual multiplication of functions.

(b) $C_{\kappa}$ is the largest translation invariant subspace of $L_{\kappa}^{\infty}$ on which the shift operator $T_{x} f(t)=f(t-x)$ has the property $\left\|T_{x} f-f\right\|_{\kappa} \rightarrow 0$, if $x \rightarrow 0$.

(c) The polynomials are dense in $C_{\kappa}$. In particular, $C_{\kappa}$ is separable.

Proof. (a) It is enough to show that $\left(R L_{\kappa}^{\infty},\|\cdot\|_{\infty}+\|\cdot\|_{\kappa}\right)$ is a Banach algebra. Submultiplicativity of the norm $\|\cdot\|_{\infty}+\|\cdot\|_{\kappa}$ follows directly form Theorem 1 and (iii) of the Lemma. To show completeness of $R L_{\kappa}^{\infty}(T)$ take a sequence $\left\{f_{n}\right\}_{n=1}^{\infty}$ in $R L_{\kappa}^{\infty}$ which is Cauchy in the norm $\|\cdot\|_{\infty}+\|\cdot\|_{\kappa}$. If $f$ is the uniform limit of $f_{n}$ then $\Omega_{f_{m}-f_{n}}(t, s) \rightarrow \Omega_{f-f_{n}}(t, s)$ as $m \rightarrow \infty$ uniformly in $(t, s)$. By Fatou's lemma,

$$
\begin{aligned}
\left\|f-f_{n}\right\|_{\kappa} & =\int_{T \times[0,1]} \Omega_{f-f_{n}}(t, s / 2) \frac{d \mu_{\kappa}(s)}{s} d t \leqslant \frac{\lim }{m \rightarrow \infty} \int_{T \times[0,1]} \Omega_{f_{m}-f_{n}}(t, s / 2) \frac{d \mu_{\kappa}(s)}{s} d t \\
& =\lim _{m \rightarrow \infty}\left\|f_{m}-f_{n}\right\|_{\kappa}
\end{aligned}
$$

which shows that $\left\|f-f_{n}\right\|_{\kappa} \rightarrow 0$ as $n \rightarrow \infty$.

(b) Let $f \in R C_{\kappa}$. We must prove that $\lim _{x \rightarrow 0}\left\|T_{x} f-f\right\|_{\kappa}=0$. Pick $0<\delta<1$.

$$
\begin{aligned}
\varlimsup_{x \rightarrow 0}\left\|T_{x} f-f\right\|_{\kappa}= & \varlimsup_{x \rightarrow 0}\left(\int_{T} \int_{0}^{\delta} \Omega_{T_{x} f-f}(t, s / 2) \frac{d \mu_{\kappa}(s)}{s} d t\right) \\
& +\varlimsup_{x \rightarrow 0}\left(\int_{T} \int_{\delta}^{1} \Omega_{T_{x} f-f}(t, s / 2) \frac{d \mu_{\kappa}(s)}{s}\right) d t \\
& \leqslant \varlimsup_{x \rightarrow 0} \int_{T} \int_{0}^{\delta}\left(\Omega_{T_{x} f}+\Omega_{f}\right) \frac{d \mu_{\kappa}(s)}{s} d t+\varlimsup_{x \rightarrow 0}\left(\int_{T} \int_{\delta}^{1} \Omega_{T_{x} f-f} \frac{d \mu_{\kappa}(s)}{s} d t\right) \\
& =2 \int_{T} \int_{0}^{\delta} \Omega_{f} \frac{d \mu_{\kappa}(s)}{s} d t .
\end{aligned}
$$


By the dominated convergence theorem,

$$
\varlimsup_{x \rightarrow 0}\left\|T_{x} f-f\right\|_{\kappa} \leqslant 2 \varlimsup_{\delta \rightarrow 0} \int_{T} \int_{0}^{\delta} \Omega_{f} \frac{d \mu_{\kappa}(s)}{s} d t=0 .
$$

(c) By part (b) we have that $C_{\kappa}$ is a homogeneous Banach space on $T$ [5, Definition 2.10] and, consequently, polynomials are dense in it [5, Theorem 2.12].

REMARK. We will give the following probabilistic interpretation of the $\mu$-norms. We will pick an interval $I=\{\tau ; d(t, \tau)<s / 2\}$ in $T$; the center $t$ of $I$ is chosen with the probability evenly distributed along $T$ and the length $|I|=s$ is chosen with the probability of the distibution $\mu(s)=\int_{0}^{s} d \mu(\tau)$. The $\mu$-norm of a function $f$ on $T$ is simply the expectation of the random variable $X(I)=$ mean oscillation of $f$ on $I=\Omega_{f}(t, s / 2) / s$ in this process.

\section{REFERENCES}

1. B. Korenblum, On a class of Banach spaces of functions associated with the notion of entropy, manuscript.

2. , A generalization of two classical convergence tests for Fourier series and some new Banach spaces of functions, Bull. Amer. Math. Soc. (N.S.) 9 (1983), 215-218.

3. A. Zygmund, Trigonometric series, Cambridge Univ. Press, London and New York, 1959.

4. F. F. Bonsall and J. Duncan. Complete normed algebras, Springer-Verlag, Berlin and New York, 1973.

5. Y. Katznelson, An introduction to harmonic analysis, Wiley, New York 1968.

Department of Mathematics, State University of New York, Albany, New York 12222

Department of Mathematics, Technical University, Wroclaw, Poland 